Regards sur l'économie allemande

Bulletin économique du CIRAC

$84 \mid 2007$

Varia

\title{
Opinion : les valeurs « bourgeoises », ciment de la société allemande
}

Isabelle Bourgeois

\section{(2) OpenEdition}

1 Journals

Édition électronique

URL : http://journals.openedition.org/rea/576

DOI : 10.4000/rea.576

ISBN : 978-2-8218-0864-5

ISSN : 1965-0787

\section{Éditeur}

CIRAC

Édition imprimée

Date de publication : 1 décembre 2007

Pagination : 36-38

ISSN : 1156-8992

Référence électronique

Isabelle Bourgeois, "Opinion : les valeurs «bourgeoises », ciment de la société allemande », Regards sur l'économie allemande [En ligne], 84 | décembre 2007, mis en ligne le 01 décembre 2009, consulté le 15 septembre 2020. URL : http://journals.openedition.org/rea/576

Ce document a été généré automatiquement le 15 septembre 2020.

(c) CIRAC 


\title{
Opinion : les valeurs «bourgeoises ", ciment de la société allemande
}

\author{
Isabelle Bourgeois
}

1 Les Allemands redécouvrent les valeurs «bourgeoises" (bürgerlich) sur lesquelles ils avaient construit leur identité depuis le Siècle des Lumières. Les profondes mutations des valeurs intervenues après 1968 les avaient reléguées au musée de l'Histoire. C'est l'Institut für Demoskopie d'Allensbach (IfD) qui avait le premier révélé ces changements en 1975, choquant profondément l'opinion allemande. Le même institut constate aujourd'hui, dans sa contribution mensuelle de novembre 2007 au quotidien Frankfurter Allgemeine Zeitung (Allensbach-Analyse, 14-11-07), qu'elles ont opéré un retour en force.

\section{Les Allemands se disent « bourgeois » : $75 \%$ à l'ouest et $49 \%$ à l'est}

240 ans après mai 1968, convictions et valeurs "bourgeoises" ont retrouvé une connotation largement positive dans l'opinion, comme il ressort de la dernière enquête représentative de l'IfD, réalisée entre le 26-10 et le 8-11-2007. A l'assertion: "si quelqu'un disait de vous que vous avez des convictions bourgeoises", $56 \%$ des Allemands répliquent "je serais d'accord». Voici cinquante ans (février 1957), ils étaient encore $75 \%$. Même les Allemands de l'est semblent aujourd'hui se rallier à cette approche, puisqu'ils sont $42 \%$ à se dire d'accord. Quand on demande aux sondés : "Est-ce que vous diriez de vous-même que vous êtes bourgeois? ", $75 \%$ des Allemands de l'ouest et $49 \%$ des Allemands de l'est disent «oui».

3 Que recouvrent ces valeurs avec lesquelles les Allemands s'identifient à nouveau? Elles n'ont rien à voir avec le stéréotype du "petit-bourgeois » borné, politiquement "à droite » et plus encore égoïste, tel qu'il sous-tendait en Allemagne aussi, depuis 1968, le qualificatif de «bourgeois». Elles trouvent bien plutôt leurs racines dans ces «vertus 
bourgeoises" issues de l'Aufklärung, comme le révèle cette autre question posée aux sondés.

\begin{tabular}{|c|c|c|c|}
\hline \multicolumn{4}{|c|}{$\begin{array}{l}\text { "Quand on dit de quelqu'un qu'il a des convictions bourgeoises, qu'est-ce que cela recouvre au } \\
\text { juste? Voici quelques explications qu'on nous a données:lesquelles trouvez-vous bien et } \\
\text { pertinentes?" }\end{array}$} \\
\hline $\begin{array}{l}\text { Cela veut dire que ce } \\
\text { quelqu'un... }\end{array}$ & & $\begin{array}{l}\text { - s'engage et agit dans l'intérêt } \\
\text { collectif }\end{array}$ & $44 \%$ \\
\hline $\begin{array}{l}\text { - s'engage pour le bien-être de } \\
\text { sa famille }\end{array}$ & $62 \%$ & - est cultivé & $37 \%$ \\
\hline $\begin{array}{l}\text { - est fiable et digne de } \\
\text { confiance }\end{array}$ & $61 \%$ & $\begin{array}{l}\text { - est calme, pépère, aime son } \\
\text { confort }\end{array}$ & $18 \%$ \\
\hline - est quelqu'un de correct & $61 \%$ & - est riche, a du patrimoine & $12 \%$ \\
\hline $\begin{array}{l}\text { - est un citoyen sur qui on peut } \\
\text { compter }\end{array}$ & $60 \%$ & - est politiquement à droite & $12 \%$ \\
\hline - est pour l'ordre et la paix & $60 \%$ & - a l'esprit borné & $12 \%$ \\
\hline - est travailleur et zélé & $58 \%$ & - est arriéré, contre le progrès & $8 \%$ \\
\hline $\begin{array}{l}\text { - est économe et gère bien son } \\
\text { argent }\end{array}$ & $45 \%$ & $\begin{array}{l}\text { - a le cœur sec, ne s'intéresse pas } \\
\text { aux autres }\end{array}$ & $4 \%$ \\
\hline
\end{tabular}

Source : Institut für Demoskopie Allensbach, F.A.Z., 14-11-2007

\section{Des valeurs fortes et partagées pour l'éducation des enfants}

4 Il semblerait que ces "vertus" amorcent une renaissance depuis une vingtaine d'années, écrit Thomas Petersen, membre de cet IfD qui a fêté ses 60 ans en mai dernier et qui a joué un rôle pionnier dans l'essor, outre-Rhin, des sciences sociales théoriques et appliquées (voir REA 80/07). L'institut analyse ainsi entre autres (et depuis 1967) l'évolution des comportements éducatifs ou des valeurs qui sous-tendent l'éducation. En 1992, par exemple, 73 \% des Allemands affirmaient que les objectifs prioritaires sont d'apprendre aux enfants à " être polis et à bien se conduire "; ce taux est passé aujourd'hui à $89 \%$. "Apprendre à faire bien et consciencieusement son travail » recueille désormais $78 \%$ des suffrages (contre $69 \%$ voici quinze ans). Autres vertus revalorisées : "être économe » (le taux de réponses est passé de $59 \%$ à $70 \%$ ) et "avoir soif d'apprendre " (de $49 \%$ à $68 \%)$. Comme on pouvait s'y attendre, ces priorités éducatives sont particulièrement nettes chez ceux qui s'affirment eux-mêmes «bourgeois ». Les mêmes sont particulièrement férus de culture et engagés dans la vie sociale, rappelle l'analyse. 


\section{Le sens complexe de bürgerlich : vertus bourgeoises et citoyenneté}

5 Les Allemands se sentent donc foncièrement «bourgeois». Mais attention! Il faut se garder de confondre le sens accordé outre-Rhin à "bürgerlich" et sa traduction française par «bourgeois» - faute de mieux. Car il s'agit d'un 'faux ami' par excellence. Bürgerlich, depuis la reconstruction d'une République fédérale démocratique et prospère au sortir de la guerre, signifie avant tout «comme il faut ». La connotation est donc forcément positive, qu'il s'agisse des valeurs "communément partagées» (bürgerliche Werte), des partis politiques "établis ", c'est-à-dire non extrémistes, donc « du milieu » (bürgerliche Parteien), ou tout simplement de la «bonne cuisine familiale » (gut bürgerliche Küche). A cette première acception générale s'en ajoute une autre, politique (mais non politicienne!), qui désigne ce qu'il conviendrait d'appeler en français la «classe moyenne » (bürgerliche Gesellschaft) ou plutôt : cette large base de la société qui n'appartient ni à la noblesse ni au 'prolétariat'. Elle se conjugue indissociablement en RFA avec la notion de « citoyen » (Staatsbürger). Autrement dit : le qualificatif bürgerlich renvoie aussi bien à des valeurs qu'à la citoyenneté et, dans la mesure où le principe de base sur lequel repose l'organisation de la société allemande au sens large du terme est la subsidiarité, il signifie, appliqué à l'individu, que celui-ci se considère comme un acteur impliqué dans un jeu collectif et responsable de ses actes envers la communauté (famille, amis, entreprise, Etat...). La "société bourgeoise » (Bürgergesellschaft) est donc la « société civile » au sens plein du terme.

6 Cette acception large et consensuelle du qualificatif de bürgerlich a connu deux ruptures dans l'histoire récente. La première est son usage idéologique, réducteur et péjoratif, fait par les régimes communistes (RDA en tête) pour désigner " l'adversaire de classe " du temps de la partition du monde. La seconde est son usage tout aussi partisan fait par les mouvements d'extrême gauche et leurs émules à partir de 1968 pour se démarquer des " profiteurs du système » en général et - singularité allemande - de la génération précédente en particulier, accusée d'avoir "pactisé » avec le régime hitlérien. Cette double rupture, conjuguée à la tendance générale, européenne et mondiale, à la diversification des modes de vie et des valeurs (voir REA 59/02), contribue à expliquer pourquoi les valeurs fondatrices de la société allemande s'étaient longtemps trouvées comme marginalisées, voire refoulées.

\section{Un retour aux sources...}

7 Dans un monde globalisé, en constant changement, le retour aux sources et aux valeurs sûres offre le seul cadre de référence encore stable. L'analyse de l'IfD confirme cette tendance, révélée par tant d'autres sondages. Lorsqu'on leur a demandé quels termes ils associent avec le qualificatif de "bürgerlich», les Allemands ont répondu: «tradition» (83\%), «famille» (83\%), «bienséance et bonnes manières» (71\%), « conservateur » (63\%), « faire des économies » $(62 \%)$, « responsabilité » $(60 \%) .$. 


\section{... qui pourrait se traduire en politique}

8 Cette tendance, même partielle, aux retour aux sources "pourrait à long terme avoir un impact sur le rapport de forces politique ", en conclut l'IfD. Certes, $47 \%$ des Allemands préfèrent appliquer le qualificatif de « bourgeois » à la CDU/CSU plutôt qu'au SPD ou au FDP ( $28 \%$ ex aequo) ; quant aux Verts, ils ne rapportent que $9 \%$ des suffrages. Bien entendu, les partisans ou membres d'un de ces partis accordent majoritairement ce qualificatif au leur. Cela dit, à la question : "Quand on dit d'un parti qu'il est bürgerlich, est-ce que cela plaide plutôt pour ou contre ce parti ?", les Allemands sont 58 \% à répondre "pour ». La conclusion qu'en tire l'Institut d'Allensbach - à la veille, rappelons-le, des congrès des partis CDU et SPD (voir dans ce numéro) : le parti politique "qui se reconnaît ouvertement bürgerlich ne passera peut-être pas pour l'avant-garde, mais pourra être certain d'être compris de la majorité des Allemands ». Les prochaines élections au Bundestag (2009) confirmeront ou infirmeront cette tendance. En attendant, la CDU, si elle évite encore l'adjectif bürgerlich dans son nouveau programme, a mis en avant un autre de ses synonymes : «Die Mitte» - le centre ou, plutôt : le «milieu ». Un emploi qui fait suite à ce « nouveau milieu » (Neue Mitte) prôné par le SPD en 1998.

INDEX

Mots-clés : valeur, société 